\title{
Aristoteles und die Ordnung der Dinge bei Fray Luis de Granada, Francisco Sánchez, Juan Huarte und Antonio de Torquemada
}

Strosetzki, Christoph

First published in:

Der Prozeß der Imagination, S. 63 - 79, Max Niemeyer Verlag, Tübingen 2005 ISBN 3-484-52921-0

Münstersches Informations- und Archivsystem multimedialer Inhalte (MIAMI) URN: urn:nbn:de:hbz:6-52439460084 
Aristoteles und die Ordnung der Dinge bei Fray Luis de Granada, Francisco Sánchez, Juan Huarte und Antonio de Torquemada

Bockhirsch [griech.: tragélaphos] oder Sphinx [griech.: Sphígx] entsprechen nicht der normalen lebensweltlichen Ordnung, da sie als Fabelwesen in der menschlichen Phantasie, nicht aber in der physischen Realität anzutreffen sind. Da nach Aristoteles von dem, was ist, alle annehmen, daß es irgendwo ist, das jedoch, was es nicht gibt, sei auch nirgendwo, stellt er zu Recht die Frage, wo es denn Bockhirsch oder Sphinx gebe. ${ }^{1}$ Während er diese Frage offen lässt, deutet er an zwei weiteren Stellen seiner Physik Alternativen an, die das Verständnis seiner Lehre von den vier Ursachen voraussetzen, nach der jedes Ding nicht nur eine kausale, sondern auch eine materielle, formale und zielorientierte Ursache hat. So sei das fertige Haus kausal verursacht durch die Arbeit der Handwerker, materiell durch die Baustoffe, formal durch die Baupläne des Architekten und schließlich zielorientiert durch die Vorstellung des Architekten vom zu erreichenden Endprodukt. Da nun Bockhirsch oder Sphinx keinen Platz im alltäglichen Ordnungssystem haben, gibt es zwei Möglichkeiten: Entweder sie sind wie die ungeordneten Materialien auf der Baustelle noch auf dem Weg, sich gemäß der Zielursache aus Ungefügtem zu Wohlgefügtem zu entwickeln und markieren damit eine Zwischenstufe hin zum Endprodukt. Oder es handelt sich um durch irgendetwas bedingte Mißbildungen, wie bei der falschen Verabreichung eines Heilmittels durch den Arzt. In diesem Fall waren sie nicht in der Lage zu einem Ziel oder Schluß zu gelangen, da sie durch materiell bzw. formal entgegenstehende Ursachen an ihrer vollen Entwicklung gehindert wurden. Im ersten Fall handelt es sich um Zwischenstufen einer noch fortschreitenden Realisierung, im zweiten um verirrte Fehlkonstruktionen. ${ }^{2}$ Denn - so meint Aristoteles: "Bei den Naturabläufen gilt [...] das 'immer gleich', außer wenn etwas störend dazwischentritt."

Da bei Aristoteles die Zielursache und nicht die Wirkursache im Mittelpunkt steht, lehnt er konsequent in seiner Auseinandersetzung mit Empedokles ${ }^{4}$ eine Beschränkung auf Wirkursachen, wie sie später Darwins Evolutionstheorie formuliert, ab, da z.B. "Rindsgattungen mit Mannsbug" oder "Weinstockartiges mit Olivenbaumspitzen" nicht durch Weiterentwicklung, sondern durch Störung der Naturanlage entstehen, nach der alles von einem ursprünglichen Antrieb in sich selbst bei fortlaufender Veränderung zu einem bestimmten Ziel gelangt. ${ }^{5}$ Allerdings beschäftigt sich Aristoteles nicht in erster Linie mit

1 Aristoteles, Physik, in: ders., Philosophische Schriften, 6 Bde., übers. v. Hans Günter Zekl, Hamburg 1995 (Meiner), Bd. 6, S. 74; wegen unterschiedlicher Übersetzungen und Einleitungen werden bewußt verschiedene Aristotelesausgaben benutzt.

2 Vgl. ebenda, S. 13, $45 \mathrm{f}$.

3 Ebenda, S. 46.

4 Vgl. ebenda, S. 35-39.

5 Vgl. Aristoteles, Physik (Anm. 1), S. 46. Zufall ist bei Aristoteles das Resultat einer NebenbeiUrsache: z.B. wenn ein Pferd zufällig herauskam und es deshalb einem Unglück entging, aber nicht herauskam in der Absicht, diesem Unglück zu entgehen (vgl. ebenda, S. 40). Eine gewisse Unklarheit ergibt sich erst, wenn das von Natur Angelegte auch als Wirk- und nicht nur als 
Außergewöhnlichem. ${ }^{6} \mathrm{Da}$ er es aber in das System der alltäglichen Dinge integriert, hat man eben dort weiter zu suchen. Im Folgenden gehe ich daher zunächst auf Aristoteles und seine Prinzipien der Anordnung von gewöhnlicher und außergewöhnlicher Empirie ein. Es folgen Belege zur Aristotelesrezeption in der Philosophie des 16. Jahrhunderts in Spanien. Schließlich bleibt in einem dritten Teil zu zeigen, was die aristotelischen Ordnungsprinzipien für Antonio de Torquemada bedeuten. Da Michel Foucault bekanntlich ausführlich das Prinzip der Ähnlichkeit mit seiner Bedeutung für die frühe Neuzeit vorgeführt hat, gilt im Folgenden die besondere Aufmerksamkeit erstens der Anordnung von Phänomenen nach Identitäten und Unterschieden bzw. den Möglichkeiten der Klassifikation und der Taxonomie und zweitens der Betrachtung von Phänomenen in ihrer historischen Bedingtheit und Gewordenheit als Resultate von Gestaltungen, Wirkungen und Funktionen. Mit anderen Worten: Es soll gefragt werden, ob die von Foucault dem 17. und dem 19. Jahrhundert zugeordneten Ordnungsschemata bereits bei Aristoteles angelegt sind und ob sie im Kontext der Aristotelesrezeption des 16. Jahrhunderts eine Rolle spielen. ${ }^{7}$

Bekanntlich hängt auch das Interesse für Klassifikation und Funktionalisierung in der ersten Hälfte des 19. Jahrhunderts mit der Tatsache zusammen, daß für Aristoteles, gefördert durch A. Trendelenburg, ${ }^{8}$ ein neues Interesse erwachte, das z.B. in Frankreich Früchte trägt durch die Victor Cousin gewidmete Übersetzung der aristotelischen Werke durch den in der Politik und am Collège de France tätigen Jules Barthélemy Saint-Hilaire (1805-

Zielursache gesehen wird, z.B. wenn sich wie im Fall von Wasser und Luft das Leichte aufwärts und das Schwere abwärts bewegt und Aristoteles als Ursache erklärt: "Das 'dort-und-dorthin' ist ihnen naturgegeben" (ebenda, S. 203).

- Die Aristoteles wahrscheinlich zu Unrecht zugeschriebenen 'Wunderberichte' versammeln hypothesenartig Gerüchte und Berichte von außerordentlichen und erstaunlichen Phänomenen, ohne jedoch deren Wahrheitsgehalt abzuwägen. Sie werden in unserem Zusammenhang nicht berücksichtigt: vgl. Aristoteles, Mirabilia, in: ders., Werke, übers. v. Hellmut Flashar, Darmstadt 1972, Bd. 18, S. 7-36.

7 Vgl. Michel Foucault, Les mots et les choses, Paris 1966; vgl. auch Klaus W. Hempfer, "Probleme traditioneller Bestimmungen des Renaissancebegriffs und die epistemologische Wende", in: ders., Diskursstrukturen und epistemologische Voraussetzungen, Stuttgart 1993, S. 9-45; er stellt die Bedeutung der analogia entis als zentraler epistemologischer Konfiguration im 16. Jahrhundert angesichts unzureichender Belege Foucaults in Frage und fragt nach möglichen Ursprüngen im Mittelalter bzw. in der Antike. In ähnlicher Weise verändert auch B. F. Scholz Foucaults Vorgaben: Die Barockforschung, schlägt er vor, soll davon ausgehen, daß in Texten entweder die diskursive Praxis der Ähnlichkeit oder die der 'Repräsentation' präsent ist, aber auch beide zusammen dominieren können. Bernhard F. Scholz, "Zur Bedeutung von Michel Foucaults These eines epistemischen Bruchs im 17. Jahrhundert für die Barockforschung", in: Klaus Garber (Hg.), Europäische Barock-Rezeption, Wiesbaden 1991, S. 169-184.

8 Vgl. Adolf Trendelenburg, De Aristotelis categoriis prolusio academica, Berolini 1833; ders., Geschichte der Kategorienlehre, Berlin 1846; vgl. auch die zeitgenössischen einschlägigen Schriften zur aristotelischen Logik von P. Gumposch, H. Rassow und H. Hettner. Im 19. Jahrhundert erlebt das Thema 'Zweck' eine neue Blüte: Der Vitalismus des Biologen Hans Driesch verwendet sogar den Terminus Entelechie. In der Philosophie Gustav Theodor Fechners (1801-1887) und Hermann Lotzes (1817-1881) lebt der Entelechiegedanke fort. Vgl. Walther Kranz, Die griechische Philosophie, Leipzig ${ }^{2} 1986$, S. 232. 
1895). ${ }^{9}$ Die aristotelische Einteilung der Tiere in bluthabende und blutlose entspricht der Einteilung in Wirbeltiere und wirbellose Tiere, die der französischen Naturforscher Georges Baron de Cuvier 1817 vorgenommen hat. Die blutlosen Tiere teilt Aristoteles in Schalentiere, Krustentiere, Weichtiere und Insekten, die Tiere mit Blut in Fische, Amphibien, Vögel und Säugetiere. ${ }^{10}$

Wichtiger für unseren Zusammenhang aber ist, daß zur Zeit der Renaissance nicht nur der Platonismus erstarkte, sondern die bessere Kenntnis der griechischen Sprache zahlreiche neue und bessere Ausgaben fast aller aristotelischer Werke in lateinischer und griechischer Sprache hervorbrachte. Im Trivium der sieben freien Künste und damit in der Grundausbildung eines jeden Intellektuellen hatte die Logik - es war im wesentlichen die aristotelische - einen der Grammatik und Rhetorik gleichberechtigten Platz. " Im gesamten 16. Jahrhundert war also die aristotelische Logik mit ihrem porphyrianischen Kommentar prägend und förderte Festlegungen von Identitäten, Unterschieden und Klassifikationen. Dies zeigt am deutlichsten das berühmte, das Organon des Aristoteles einleitende Werk Isagoge über die der Definition, der Begriffseinteilung und dem Beweis dienenden funf Prädikabilien Genus, Spezies, Differenz, Proprium und Akzidens des Neuplatonikers Porphyrius (233-304 n.Chr.), das " $z u$ den gelesensten und verbreitetsten Schriften unserer Culturgeschichte"12 gezählt wird. In diesem Werk kommt gerade dem von Foucault

9 Logique d'Aristote (1839-1844); La Métaphysique d'Aristote (1879); De la physique d'Aristote (1862); vgl. auch Eugène Thionville, La théorie des lieux communs dans les topiques d'Aristote et les principales modifications qu'elle a subies jusqu'à nos jours, Paris 1855.

10 Vgl. Aristoteles, Tierkunde, in: ders., Die Lehrschriften, Bd. 8,1, hg. v. Paul Gohlke, Paderborn 1949 (Schöningh). Die Einteilung der anatomischen Betrachtungen erfolgt nach dem Unterschied der Organe, deren Teile ihnen nicht selbst gleich sind, wie die Hand nicht aus Händen besteht, und dem der Substanzen, deren Teile einander gleichartig sind, wie ein Teil eines Fleischstückes wiederum Fleisch ist. Der Betrachtung der inneren Teile, der Sinne, der Zeugung und Entwicklung, schließen sich Beobachtungen über die Lebensweisen der unterschiedlichen Tiertypen an.

11 José Luis Abellán unterscheidet die "cátedra de Prima de Lógica" und die "cátedra de Vísperas": Während sich erstere auch Autoren wie Petrus Hispanus widmete, konzentrierte sich letztere auf das Organon des Aristoteles und auf seinen Kommentator Porphyrius. José Luis Abellán, Historia crítica del pensamiento español, Bd. 2, Madrid 1979, S. 546-553. In Alcalá wurde die Logik bis 1550 nach dem Vorbild der noch immer scholastisch geprägten Pariser Universität gelehrt. Die 1550 einsetzende Renaissancelogik kehrt unter dem Einfluß des Konzils von Trient zu einem thomistisch verstandenen Aristoteles zurück. Sehr genau schildert Wilhelm Risse das komplexe Beziehungsgeflecht von traditionellem Thomismus, von unter Pariser Einfluß stehender Neuscholastik, von Humanismus und von jesuitischer Gegenreformation in der spanischen Rezeption aristotelischer Logik (Logik der Neuzeit, Bd. 1 [1500-1640], Stuttgart/Bad Cannstatt 1964, S. 308-439).

12 Carl Prantl, Geschichte der Logik im Abendland, 4 Bde., Leipzig 1855-1870, Bd. 1, S. 626. Weitere beliebte Kommentare zur aristotelischen Logik waren der Pseudo-Aristoteles und der Pseudo-Platon. Während ersterer einzelne Begriffe systematisch in baumstrukturige Filiationen aufteilt, bemüht sich letzterer um Definitionen wichtiger Begriffe. Vgl. Aristoteles, Organon [Griechisch-Deutsch], hg. v. Hans Günter Zekl, Hamburg 1998 (Meiner), Bd. 2, S. 189-231, 233245. 
hervorgehobenen Begriff der Differenz ${ }^{13}$ eine zentrale Rolle zu. ${ }^{14}$ Bei Einteilungen und Definitionen sei nämlich vorzugehen nach Gattung, z. B. Sinnenwesen oder animalisches Wesen, Art ("Mensch"), Differenz ("vernünftig"), Proprium ("was die Fähigkeit hat zu lachen") und Akzidenz ("weiß, schwarz, sitzen"). ${ }^{15}$ Nach Porphyrius würde eine pyramidenartige Anordnung der Gegenstände nach den genannten Kriterien z. B. mit dem Seienden das Generellste und am anderen Ende mit Individuen, wie Sokrates oder Platon, das Speziellste bilden.

Die Differenz kann nach dem Kommentar des Porphyrius allgemein, eigentlich oder im eigentlichsten Sinn sein. Allgemein unterscheidet sich das eine vom anderen oder von sich selbst durch irgendeine Verschiedenheit, wie z.B. Sokrates von Plato oder der eine von sich als Kind und als Erwachsener oder in unterschiedlichen Zuständlichkeiten wie tätig oder ruhend. Ein eigentlicher Unterschied ist durch ein untrennbares Akzidenz wie Blauäugigkeit oder Krummnasigkeit gegeben. Der eigentlichste Unterschied ist der spezifische bzw. artbildende, z.B. wenn sich der Mensch vom Pferd durch die "differentia specifica" der Vernünftigkeit unterscheidet. Er macht im Gegensatz zu "tätig" oder "ruhend" etwas nicht nur anders beschaffen, sondern zu einer Art. Hier gilt: "Differenz ist, um was die Art reicher ist als die Gattung. Denn Mensch ist um die Momente vernünftig und sterblich reicher als Sinnenwesen." ${ }^{16}$ Aristoteles präzisiert: "Was in verschiedenen Gattungen steht, ohne sich untergeordnet zu sein, hat auch der Art nach verschiedene Differenzen, wie z.B. Sinnenwesen und Wissenschaft. Die Differenzen von Sinnenwesen sind Gangtier, Zweifüßler, Flugtier, Wassertier; von diesen Differenzen gilt aber keine für die Wissenschaft; denn eine Wissenschaft unterscheidet sich nicht dadurch von der anderen, daß sie zwei Füße hat. Dagegen können sich untergeordnete Gattungen ganz wohl dieselben spezifischen Unterschiede haben."17

Spezifischer Differenz und Art ist gemeinsam, daß man an ihnen gleichermaßen teilnimmt: Sokrates ist immer vernunftbegabt und ist immer Mensch. Während die Differenz oft von mehreren Arten gilt, wie vernünftig von Engel und Mensch, trifft das Proprium nur bei der einen Art zu, deren Proprium es ist. ${ }^{18}$ Akzidenz ist das, was auftritt und verschwin-

13 “Le semblable, après s'être analysé selon l'unité et les rapports d'égalité ou d'inégalité, s'anlyse selon l'identité évidente et les différences : différences qui peuvent être pensées dans l'ordre des inférences" (Foucault, Les mots et les choses [Anm. 7], S. 68); "discerner : c'est-à-dire à établir les identités, puis la nécessité du passage à tous les degrés qui s'en éloignent" (ebenda, S. 69). Vgl. im Folgenden: Porphyrius, "Einleitung in die Kategorien", in: Aristoteles, Philosophische Schriften (Anm. 1), Bd. 1, S. 1-23. Ebenda, S. 3.

16 Ebenda, S. 11. Porphyrius unterscheidet des weiteren trennbare Differenzen, wie gesund oder krummnasig, von untrennbaren, wie vernünftig oder unvernünftig. Untrennbare haften ihrem Träger an sich an, vervollständigen den jeweiligen Wesensbegriff und dulden keine Abschwächung oder Steigerung im Gegensatz zu mitfolgend (per accidens) untrennbaren Differenzen. Aristoteles, Organon I, in: ders., Philosophische Schriften (Anm. 1), Bd. 1, S. 2-3.

18 "Die Art unterscheidet sich vom Proprium dadurch, daß die Art auch Gattung von anderem, das Proprium aber nicht Proprium von noch anderem sein kann. Und die Art ist vor dem Proprium da, das Proprium kommt zur Art hinzu: Mensch muß sein, ehevor des Lachens fähig ist" (Porphyrius, "Einlcitung in die Kategorien" [Anm. 14], S. 21). 
det ohne Untergang des Dings. ${ }^{19}$ Mögliche Akzidentien sind nach Aristoteles: "ein Quantitatives z.B. ein zwei, ein drei Ellen Langes; ein Qualitatives z.B. ein Weißes, ein der Grammatik Kundiges; ein Relatives z.B. ein Doppeltes, Halbes, Größeres; ein Wo z.B. [auf dem Markt], im Lyzeum; ein Wann z.B. gestern, voriges Jahr; eine Lage z.B. er liegt, sitzt; ein Haben z.B. er ist beschuht, bewaffnet; ein Wirken z.B. er schneidet, brennt; ein Leiden z.B. er wird geschnitten, gebrannt." ${ }^{20}$ Als Substanzen schließlich will Aristoteles die Arten und Gattungen bezeichnet wissen. ${ }^{21}$

Anliegen des Organon ist also die genaue Differenzierung, Systematisierung und Hierarchisierung der Welt. Angesichts scheinbarer Ähnlichkeiten sieht Aristoteles Vorsicht geboten: Die auf Irreführung durch die Worte zurückzuführende Frage: "Was ist 'schärfer': der Schreibstift, der Wein oder der hohe Ton?", weist er zurück, da man zwar den Oberton mit seinem Nachbarton, nicht aber mit dem Wein vergleichen könne. Pferd und Hund seien vergleichbar hinsichtlich der Frage, wer von ihnen beiden weißer ist, da sich die in Frage stehende Farbe in beiden Fällen an der Körperoberfläche befinde. Man könnte sie auch hinsichtlich der Größe vergleichen. Wasser und Stimme dagegen haben keine Ähnlichkeit, die sie vergleichbar erscheinen lassen, da die Eigenschaften an einem jeweils anderen $\operatorname{sind}^{22}$

Das zweite zentrale, mit dem der Unterscheidung verbundene Prinzip bei Aristoteles ist die Veränderung mit ihren zugrundeliegenden Funktionsformen. Schon die eingangs mit Bockhirsch und Sphinx aufgeführten Beispiele zeigen, daß Aristoteles hinter den Phänomenen zeitliche Entwicklungen, Ursachenketten und Funktionen sieht, die zu ihrer Erklärung erforderlich sind. Aristoteles betont daher: "Von Veränderung und Wandel gibt es so viele Formen wie von "seiend'."23 Er nennt Eigenschaftsveränderung, Wachsen, Schwinden, Werden, Vergehen und Ortsbewegung. Das eine ist der Wirklichkeit nach da, z.B. warm, das andere der Möglichkeit nach, z.B. kalt. Alles ist "zugleich Wirkung ausübend und Wirkung erfahrend." Und "Zur-Wirklichkeit-Kommen des Möglichen, insofern es möglich ist, das ist ganz offenkundig: Veränderung." ${ }^{24}$ Bewegung, Wandel, Prozessualität, Veränderung in allen ihren Formen werden daher in der philosophiegeschichtlichen For-

19 Proprium und untrennbares Akzidenz unterscheiden sich dadurch, daß das Proprium nur einer Art zuzuordnen ist, wie die Fähigkeit zu lachen dem Menschen, während ein untrennbares Akzidenz wie schwarz nicht nur dem Raben, sondern auch der Kohle und anderen Dingen zuzuordnen ist. Während das Schwarzsein des Raben ein untrennbares Akzidenz ist, ist das Schlafen ein trennbares.

Aristoteles, Organon I (Anm. 17), S. 3.

${ }^{21}$ Bejahung oder Vemeinung kommen erst durch die Verbindung der Begriffe zustande und sind entweder wahr oder falsch.

22 Aristoteles, Physik (Anm. 1), S 182f. Nur in einer ganz spezifischen Verwendung toleriert er Analogien: "Unter 'analog' verstehe ich z. B., daß die einen Tiere eine Lunge haben, andere aber an deren Stelle ein sonstiges Organ, oder daß die einen Blut haben, die anderen aber etwas Analoges, was für sie dieselbe Bedeutung hat wie für die Blutführenden das Blut [...]" (Aristoteles, "Zur Methodik der Naturforschung", in: ders., Hauptwerke, hg. v. Wilhelm Nestle, Stuttgart ${ }^{7} 1968$ [Kröner], S. 381). 
schung als thematisches Zentrum des Aristoteles gesehen. ${ }^{25}$ Die Erscheinungsformen sind zahlreich: Veränderungen sind für Aristoteles erklärbar als Veränderung des Was, Worin und Wann bzw. des Wiebeschaffen, des Wieviel, des Orts. ${ }^{26}$

Die allgemeinste Veränderung ist für Aristoteles die Ortsveränderung. Er nennt sie Bewegung, hebt hervor, daß der Ort nicht untergeht, wenn die in ihm befindlichen Gegenstände vergehen, und hält es für unmöglich, daß der Ort selbst ein Körper sein könnte, da ja dann zwei Körper an demselben Platz wären. ${ }^{27}$ Mit dem Ort ist die Zeit verbunden. ${ }^{28}$ Den Eindruck, es vergehe keine Zeit, tritt nach Aristoteles auf, wenn wir keine Veränderung bemerken und das Bewußtsein in einem einzigen Jetzt zu bleiben scheint. Da wir dagegen, wenn wir Veränderung wahrnehmen, sagen, es sei Zeit vergangen, ist für Aristoteles klar, $\mathrm{da} ß$ ohne wahrgenommene Bewegung und Veränderung Zeit nicht existiert. ${ }^{29}$ Wie das Beispiel der Uhr verdeutlicht, wird mit Zeit Bewegung und mit Bewegung Zeit gemessen. Entstehen, Vergehen, Wachsen, Eigenschaftsveränderung und Ortsbewegung sind Bewegungen, für deren Zeit eine Zahl angegeben werden kann. Die Zeit macht aus der eingangs erwähnten Vierursachenlehre einen Prozeß: "Eines ist das eigentlich 'in Bewegung Setzende', ein anderes das 'in Bewegung Gesetzte', - außerdem (gibt es noch) das 'in welchem' (dabei), die Zeit, und neben all dem noch das 'aus welchem' und das 'wozuhin': Jede Veränderung geht von etwas aus und zu etwas hin." 30

25 Vgl. Hans Günter Zekl, "Einleitung", in: Aristoteles, Physik [Griechisch-Deutsch], hg. v. Hans Günter Zekl, Hamburg 1987 (Meiner), Bd. 1, S. XVII. Vgl. auch Walter Bröcker, Aristoteles, Frankfurt/M. ${ }^{3} 1964$ [1935].

26 Alle ortsbezogenen Bewegungen führt Aristoteles auf Zug, Stoß, Mitnahme und Drehung zurück, wobei Einatmen ein Zug, Ausatmen aber genauso wie Spucken ein Stoß ist. "Es entsteht das im einfachen Sinn Werdende teils durch Umformung, z.B. ein Standbild; teils durch Hinzutun, z.B. Dinge, die wachsen; teils durch Fortnehmen, z.B. wenn aus dem Stein eine Hermesfigur wird; teils durch Zusammenfügung, z.B. ein Haus; teils durch Eigenschaftsveränderung, z.B. bei Dingen, die sich in ihrem Stoff wandeln. Es ist also aus dem Gesagten klar, daß jedes Werdende immer ein Zusammengesetztes ist: es gibt das Etwas, das da wird, und das, wozu dieses wird" (Aristoteles, Physik, [Anm. 1], S. 18f.).

27 Ebenda, S. 76.

28 "Da aber die Zeit in besonderem Maße eine Art Bewegung zu sein scheint und Wandel, so wäre dies zu prüfen: Die verändernde Bewegung eines jeden (Gegenstandes) findet statt an dem SichVerändernden allein oder dort, wo das in ablaufender Veränderung Befindliche selbst gerade ist; die Zeit dagegen ist in gleicher Weise sowohl überall als auch bei allen (Dingen)" (ebenda, S. $103 \mathrm{f}$.).

29 "Und wir sagen dann, daß Zeit vergangen sei, wenn wir von einem 'davor' und einem 'danach' bei der Bewegung Wahrnehmung gewinnen. [...] Was nämlich begrenzt ist durch ein Jetzt, das ist offenbar Zeit. [...] Denn eben das ist Zeit: Die Meßzahl von Bewegung hinsichtlich des 'davor' und 'danach'. [...] eine Art Zahl ist also die Zeit. [...] Womit wir zählen und das Gezählte sind aber verschieden" (ebenda, S. 105f.). Daß hier Aristoteles bereits in Ansätzen eine Vorstellung von Zeit als Anschauungsform im Sinne von Kant vertritt, sei nur erwähnt, nicht weiter vertieft (vgl. auch ebenda, S. 116, zur Bedeutung des Bewußtseins). Entsprechendes gilt auch für den Raum, wenn Aristoteles ausführt, daß ein Weg lang erscheint, wenn die Reise, d.h. die Bewegung des Subjekts auf ihm, lang erscheint.

30 Aristoteles, Physik (Anm. 1), S. 119. Handeln und Sich-Rühren ist erforderlich, wenn etwas Neues entstehen soll, dagegen "Verkommen geschieht auch ohne Rührigsein" (ebenda, S. 115). Zcit wird zum Grundprinzip der Vergänglichkeit, da etwas, das zu einer Zeit ist, zur anderen 
Nur einzelne Beispiele für die verbreitete Aristotelesrezeption in der Philosophie des 16. Jahrhunderts in Spanien seien herausgegriffen. Fray Luis de Granada soll für die zahlreichen theologisch geprägten Autoren stehen. Sein "orden de las cosas" differenziert zwischen den vier Elementen als einfachen Dingen mit nicht mehr als zwei Eigenschaften, den gemischten wie Schnee, Regen, Wind, denen eine zusätzliche Eigenschaft zukommt, denjenigen wie Steinen, Perlen und Metallen, denen eine klare Form zukommt, denjenigen, die zusätzlich noch über Leben verfügen und die wachsen, wie Bäume und Pflanzen, dann den Tieren, die zwar Sinnesorgane aber kein Bewegungsvermögen haben wie Austern oder Muscheln, weiterhin den Tieren, die wie Vögel oder Fische zudem das Bewegungsvermögen haben, und dem Menschen, der zusätzlich zu allen genannten Eigenschaften auch noch den Verstand hat, durch den er sich von allen anderen unterscheidet. ${ }^{31}$ Betrachtet man nun die Gattung der Tiere näher, dann lassen sich nach Luis de Granada Arten aus folgenden Unterschieden ableiten: Die einen suchen ihre Nahrung beim Laufen, andere durch die Erde kriechend, andere fliegend, wieder andere schwimmend; es gibt solche, die die Nahrung mit den Zähnen und dem Mund nehmen, andere zerstückeln sie mit den Krallen, wieder andere mit dem erhobenen Schnabel, die einen saugen, die anderen benützen die Hände ${ }^{32}$ Die "maravillas" der Welt zeigten sich nicht in einer Tiergattung, sondern in allen und auf unterschiedlichste Weise, "que ningunas escrituras hasta agora las han podido comprehender, mayormente que cada día en nuevas tierras se descubren nuevos animales y nuevas habilidades y propiedades de ellos, que nunca en estas nuestras tierras han sido conocidas." ${ }^{33}$ Bei allem Bestreben, das Bekannte zu klassifizieren, bleibt Neugier für die Einordnung des Unbekannten.

Von Aristoteles, allerdings indirekt über Thomas von Aquin, übernimmt Fray Luis de Granada auch die Vorstellung von der beständigen Bewegung der Dinge, die entweder in sich oder außerhalb ihrer die Ursache dafür haben. So bringt die Seele den Körper in Bewegung, verläßt ihn die Seele, fehlt ihm auch die von ihr verursachte Bewegung. ${ }^{34}$ Letzter, seinerseits unbewegter Beweger, Ursache aller Ursachen, ist Gott. ${ }^{35}$ Der Mensch

nicht, als in der Zeit stehend gedacht werden muß. Vergehen ist Wechsel zum Nichtsein, Werden Wechsel zum Sein (vgl. ebenda, S. 119).

31 Fray Luis de Granada, Introducción del símbolo de la fe I, in: ders., Obras Completas, hg. v. Alvaro Huerga, Madrid 1996 (Fundación Universitaria Española, Dominicos de Andalucía), Bd. 9, S. 48-49. Vgl. ebenda, S. 64.

33 Ebenda, S. 129.

34 Ebenda, S. 49.

35 "Pues este cielo, según lo presupuesto, ha de tener movedor que lo mueva: Pues de este movedor se pregunta si en su ser y en la virtud que tiene para causar este movimiento, tiene dependencia de otro, o no: si no la tiene, sino por sí mismo tiene su ser y su poder, ese tal llamaremos Dios, porque sólo Dios es el que, como superior de todas las cosas, no pende, ni en su ser ni en su poder, de nadie, sino de sí mismo. Mas si me decís que tiene otro superior, de quien depende cuanto al ser y cuanto a la virtud del mover, dese superior haré la misma pregunta que del inferior: y procediendo en este discurso, o se ha de dar proceso en infinito, lo cual dijimos ser imposible, o habemos finalmente de venir a un primer movedor, de que penden los otros movedores, y a una primera causa, de cuya virtud participan su virtud todas las otras causas, y ésa es a quien llamamos Dios" (Fray Luis, Introducción [Anm. 31], S. 49-50). "Porque dado caso que Dios sea la primera causa que mueve todas las otras causas, pero estos cuerpos con las 
schließlich ist die Zweckursache der Schöpfung. ${ }^{36}$ Die Teile des menschlichen Körpers haben die Funktion bzw. Zielursache, optimal die Aufrechterhaltung des Lebens zu garantieren. ${ }^{37}$ Entsprechendes gilt für die Fähigkeiten der Tiere, deren weitere Zielursache die Bewahrung des Lebens bzw. der Art ist. Es zeigt sich, daß Granadas "orden de las cosas" nicht nur bei der Differenzierung von Arten und Gattungen, sondern auch bei der Bedeutung von Wirkursachen und Funktionen bzw. Zielursachen deutlich aristotelischen Ordnungsschemata verpflichtet ist.

Ángesichts der Bedeutung des aristotelischen Paradigmas wird erklärlich, daß der Angriff der wenigen zeitgenössischen spanischen Skeptiker auf Wissen und Wissenschaft sich in erster Linie gegen Aristoteles richtete. Francisco Sánchez bezweifelt daher die Möglichkeit der Erkenntnis der Dinge durch ihre Ursachen: "Una vez más: ¿qué es saber? Conocer una cosa por sus causas, dicen [...] ¿Es necesario conocer todas las causas para conocer las cosas?" 38 Wolle man eine Wirkursache vollständig kennen, dann müßte man auch deren Wirkursache kennen und die weiteren vorausgehenden Wirkursachen. Angesichts der Verquickungen der Dinge ist eine umfassende Kenntnis Voraussetzung einer Einzelerkenntnis. ${ }^{39}$ Die Probleme beginnen für Francisco Sánchez allerdings schon bei der aristotelischen Definition, die auf unklare Wörter angewiesen ist: "Dirás que defines la cosa que es el hombre, con esta definición: 'animal racional mortal'. Lo niego, pues yo dudo también de la palabra 'animal', así como de 'racional' y de la otra. Seguirás definiendo éstas mediante los generos superiores y diferencias, como tú los llamas, hasta el 'Ente', que tampoco sabes lo que significa."40 "Toda la lógica de Aristóteles está llena de estas cosas", womit er die bloßen Wörter meint, "y mucho más las Dialécticas que tras él escribieron los más modernos. En efecto, a los nombres más comunes los llaman géneros, y

inteligencias que los mueven, son los principales instrumentos de que él se sirve para el gobierno de este mundo inferior, el cual de tal manera pende del movimiento de los cielos, que vienen a decir los filósofos que si este movimiento parase, todo otro movimiento cesara [...]" (ebenda, S. 69).

36 "De lo cual resulta esta armonía del mundo, compuesta de infinita variedad de cosas, reducidas a esta unidad susodicha, que es el servicio del hombre. Pongamos ejemplo comenzando del mismo hombre, el cual, según Aristóteles dice, es como fin, para cuyo servicio la divina Providencia diputó todas las cosas de este mundo inferior" (ebenda, S. 57).

37 " [...] cada una de estas partes sirve tan perfectamente, a lo que conviene a la conservación de la vida humana, que es para la sustentación de nuestro cuerpo y para el uso y oficio de los sentidos, que ningún entendimiento humano podrá descubrir en tanta variedad y muchedumbre de partes alguna cosa que falte, o que sobre, o que no venga tan a propósito de lo que es necesario para este fin, que por ninguna vía se pueda trazar otra mejor" (ebenda, S. 62).

38 Francisco Sánchez, Que nada se sabe, hg. v. Fernando A. Palacios, Madrid 1991 (Espasa Calpe, Austral), S. 77.

39 "Para saber algo perfectamente, es necesario conocer también aquello, a saber, qué cosas causan a qué otras y de qué modo. Pero hay tal concatenación entre todas las cosas que ninguna está ociosa, sino que más bien, se opone o favorece a otra; más aún, la misma cosa está destinada no sólo a perjudicar a muchas, sino también a ayudar a muchas otras. De aquí sigue que, para el perfecto conocimiento de una sola, hay que conocerlas todas" (ebenda, S. 87).

40 Sánchez, Que nada se sabe (Anm. 38), S. 57. 
a los otros especies, diferencias, propios, individuos." ${ }^{41}$ Er kommt zum Schluß: "Toda ciencia es ficción." 42

Diese pointierte Kritik ist gerichtet an alle, die wie die im folgenden vorgestellten Juan Huarte de San Juan und Juan Ginés de Sepúlveda, bei ihren Unterscheidungen verschiedener Arten von Menschen ganz selbstverständlich von Aristoteles ausgehen. Bevor Huarte die Arten von Menschen je nach ihrer Eignung für Berufe unterscheidet, geht er explizit vom aristotelischen Naturverständnis aus und postuliert eine letzte Ursache, deren Wirkungen dem Arterhalt dienen. ${ }^{43}$ Der Begriff Natur kann sich aber statt auf die Ordnung des ganzen Universums auch auf die Substanz einer einzelnen Sache beziehen, so daß die Vernunftbegabtheit in der Natur des Menschen liegt. ${ }^{44}$ Wie Fray Luis de Granada unterscheidet auch Huarte beim Menschen das Vegetative, Sensitive und Rationale. ${ }^{45}$ Er betont, "de solas tres cualidades, calor, humedad y sequedad, salen todas las diferencias de ingenios que hay en el hombre". ${ }^{46}$ Man sehe dies z.B. schon bei Aristoteles belegt, nach dem diejenigen, die in kälteren Klimazonen wohnen, wie Deutsche oder Franzosen, weniger Verstand hätten als die in wärmeren Zonen Lebenden. ${ }^{47}$ Die Unterschiede zwischen den Menschen ergeben sich also aus den jeweiligen quantitativ unterschiedlichen Anteilen der drei Komponenten Wärme, Feuchtigkeit und Trockenheit, wobei er auf Galen und Aristoteles zurückgreift. ${ }^{48}$

41 Sánchez, Que nada se sabe (Anm. 38), S. 62. Vgl. ebenda, S. 82f.: "Evidentemente: la ciencia se obtiene por demostración; ésta supone la definición, y las definiciones no pueden probarse, sino que deben ser creídas; luego la demostración a partir de suposiciones producirá una ciencia hipotética, no segura y cierta. Todo esto es concluyente desde tu posición. Además, en toda ciencia, según tú, hay que suponer unos principios, y no le corresponde a ella discutirlos; luego lo que sigue de éstos será supuesto, no sabido".

Ebenda, S. 82.

"Porque, cuando dijo Aristóteles Deus et natura nihil faciunt frustra, no entendió que Naturaleza fuese alguna causa universal, con jurisdicción apartada de Dios, sino que es nombre del orden y concierto que Dios tiene puesto en la compostura del mundo para que sucedan los efectos que son necesarios para su conservación" (Huarte de San Juan, Examen de ingenios, hg. v. Guillermo Serés, Madrid 1989 [Cátedra], S. 240-241).

44 “Aristóteles y los demás filósofos naturales descienden más en particular, y llaman naturaleza a cualquier forma substancial que da ser a la cosa y es principio de todas sus obras. En la cual significación, nuestra ánima racional con razón se llamará Naturaleza, porque de ella recebimos el ser formal que tenemos de hombres y ella mesma es principio de cuanto hacemos y obramos" (ebenda, S. 243).

Wie bei den Tieren die verschiedene Ausbildung der Fähigkeit der Augen, Ohren, des Geschmacks- und Geruchsinns zu einem unterschiedlichen Wirkungskreis führt, "porque el instrumento determina y modifica la potencia para una acción y no más", so ist es auch beim Menschen (ebenda, S. 321, vgl. auch S. 303-304).

Ebenda, S. 321.

"La mesma sentencia trae Aristóteles preguntando por qué los que habitan tierras muy frías son de menos entendimiento que los que nacen en las más calientes; y en la respuesta trata muy mal a los flamencos, alemanes, ingleses y franceses, diciendo que su ingenio es como el de los borrachos, por la cual razón no pueden inquirir, ni saber la naturaleza de las cosas. Y la causa de esto es la mucha humidad que tienen en el cerebro y en las demás partes del cuerpo" (ebenda, S. 415).

Vgl. z.B. Huarte de San Juan, Examen (Anm. 43), S. 327ff. 
Diese Komponenten sind zugleich Ursachen für unterschiedliche Arten von Menschen, ${ }^{49} \mathrm{da}$ von ihnen die unterschiedlichen Ausprägungen von Erinnerungsvermögen, Verstand und Vorstellungskraft abhängen, wodurch für verschiedene Berufe und Wissensdisziplinen geeignete Menschenarten differenziert werden. Der Gedächtnistyp eignet sich für Grammatik und Sprachen, für Rechtstheorie, Theologie, Kosmographie und Arithmetik, der Verstandesmensch für Scholastik, theoretische Medizin, Dialektik, Moralphilosophie und Justiz. Der dritte, durch Vorstellungskraft dominierte Typ schließlich sei durch Form, Korrespondenzen, Harmonie und Proportion geprägt und und fähig z.B. zur Dichtkunst, Redekunst, Staatskunst, Malerei, Schriftstellerei und Höflichkeit. ${ }^{50}$

Während Huarte die Arten von Menschen nach ihren Fähigkeiten und Tätigkeiten unterscheidet, beschäftigt sich Ginés de Sepúlveda mit dem Verhältnis von Herrschenden und Beherrschten. Dabei zitiert er Aristoteles ausgehend von Material- und Formursache fast wörtlich. Da nämlich immer die Materie der Form unterworfen ist, gibt es eine "ley divina y natural según la cual las cosas más perfectas y mejores mantienen su dominio sobre las imperfectas y desiguales." ${ }^{, 51}$ Wie sich die Haustiere in ihrem eigenen Interesse dem Menschen anvertrauen und unterwerfen, so auch die Ehefrau dem Ehemann, das Kind gegenüber dem Erwachsenen: "en una palabra, los superiores y más perfectos sobre los inferiores y más imperfectos. Y enseñan que esta misma razón vale para los demás hombres en sus mutuas relaciones, pues de ellos hay una clase en que unos son por naturaleza señores y otros por naturaleza exclavos." $52 \mathrm{Daß}$ es Menschenarten gibt, die von Natur aus Sklaven und andere, die von Natur aus Herrscher sind, war bekanntlich ein zentrales aus Aristoteles abgeleitetes Argument, mit dem Sepúlveda gegenüber Bartolomé de las Casas die Unterwerfung der Indios rechtfertigte.

Ausführlicher sei nun auf die Einordnung der außergewöhnlichen Phänomene eingegangen, die Antonio de Torquemada in seiner Sammlung Jardin de flores curiosas in der Form eines Dialoges mehrerer Protagonisten vorstellt. Immer wieder fällt das Bemühen auf, durch beharrliches Differenzieren die zahlreichen angesprochenen, gewöhnlichen und ungewöhnlichen Phänomene zu ordnen. Betrachtet man z.B. die vielen Menschen auf der Welt, die denselben Körperaufbau und Gesichtsaufbau haben, fällt auf, "que nunca falta alguna cosa en que se diferencien y conozcan." 53 Und da es auch Unterschiede zwischen

49 "Y vemos por experiencia que un hombre entiende mejor que otro y discurre mejor. Luego ser el entendimiento potencia orgánica y estar en uno más bien dispuesta que en otro lo causa" (Huarte de San Juan, Examen (Anm. 43), S. 355-356).

"De la buena imaginativa nacen todas las artes y ciencias que consisten en figura, correspondencia, armonía y proporción. Estas son: poesía, elocuencia, música, saber predicar, la práctica de la medicina, matemáticas, astrología, gobernar una república, el arte militar, pintar, trazar, escrebir, leer, ser un hombre gracioso, apodador, polido, agudo in agilibus, y todos los ingenios y maquinamientos que fingen los artífices; y también una gracia de la cual se admira el vulgo, que es dictar a cuatro escribientes juntos materias diversas, y salir todas muy bien ordenadas" (Huarte de San Juan, Examen [Anm. 43], S. 395-396).

51 Juan Ginés de Sepúlveda, Demócrates segundo, in: ders., Obras Completas, hg. v. A. Truyol y Serra, Bd. 3, Salamanca 1997 (Ayuntamiento de Pozoblanco), S. 38-134, hier: S. 55; vgl. Aristoteles, Politica, I, 3.

52 Sepúlveda, Demócrates segundo (Anm. 51), S. 55.

53 Antonio de Torquemada, Jardin de flores curiosas, hg. v. Giovanni Allegra, Madrid 1982 (Castalia), S. 106. 
Bäumen, Pflanzen, Früchten, Gräsern und Blumen gibt, an die wir gewöhnt sind, dürften wir uns eigentlich nicht allzu sehr wundern, "cuando vieramos otras cosas que salgan algún tanto de esta orden tan concertada de naturaleza. Porque ellas no salen ni exceden de naturaleza, que la falta está en nosotros y en nuestro entendimiento y juicio, que con su torpeza no lo alcanza." 54 Was wie wundersame Vorgänge oder Monstruositäten jenseits des "orden común" ist, sollte daher einen verständigen Menschen nicht aus der Fassung bringen. Erforderlich ist es, Differenzen, Arten und Akzidentien anzugeben.

Der Gesprächspartner Luis zeigt sich offen für die unendlichen Differenzen, die auch innerhalb einer Kategorie bestehen. In Bezug auf Blumen, besonders Rosen, erkennt er, wie viele Unterarten es noch innerhalb einer Art gibt. ${ }^{55}$ Und was z.B. für die Gattung gilt, trifft auch für die Art zu. Bernardo stellt fest, da sich Tiere im allgemeinen infolge bestimmter Lockrufe versammeln, gelte dies auch für die darunter zu subsumierende Art der Vögel. ${ }^{56}$ An anderer Stelle versichert er, man habe schon doppelköpfige Lebewesen gesehen, also sei dieses Phänomen auch für "otros animales", die Menschen nämlich, wahrscheinlich. ${ }^{57}$ Hier werden Merkmale der Gattung logisch konsequent auch für die Art angenommen. Betrachtet man nun den Menschen als Gattung, dann zeigt die Beschreibung der Arten von Monstermenschen, daß diese nur in einem oder in wenigen distinktiven Merkmalen von den Arten 'normaler' Menschen abweichen. Einige haben die Augen statt auf dem Kopf auf den Schultern, andere besonders große Nasen, andere einen sehr kleinen Mund, andere riesige Ohren. Die Monstrosität ist immer erst erkennbar am Grad der Abweichung vom 'normalen' Menschen. In Georgien gebe es "cinco maneras de gentes": 58 die einen seien schwarz, die anderen weiß mit Pfauenschwanz, die dritten normal, die vierten sehr klein und mit zwei Köpfen und schließlich die fünften mit Pferdegebiß als Gesicht. Der Bericht schließt mit den Worten: "y siendo esto verdad, de maravillar es que en una misma tierra haya tantas diferencias de hombres." 59 An anderer Stelle wird bezüglich eines Meermonsters explizit betont, daß es in fast allem wie ein 'normaler' Mensch war und in nur einem distinktiven Merkmal abwich: "todos los miembros eran de hombre, aunque era de muy mayor estatua; solamente se diferenciaba en tener unas pequeñas alas."

54 Torquemada, Jardín (Anm. 53), S. 106.

55 "¡Cuántas maneras hay de ellas, con cuán varias composturas y formas y con cuán delicadas colores y matices, puestas con tan gran orden y concierto!" (ebenda, S. 103). Antonio will sogar die unterschiedlichen Arten von Zauberern differenziert wissen und trennt "encantadores" von "hechiceros". Von beiden grenzt er dann später im Anschluß an Fray Alonso de Castros Buch De justa punitione hereticorum die Hexen und Hexer ab. "Los diferencia de los encantadores y hechiceros, diciendo que este linaje de hombres y de mujeres solamente se conciertan con el demonio para gozar en esta vida de todos los deleites y placeres que pueden" (ebenda, S. 314). Vgl. ebenda, S. $436 f$.

Ebenda, S. 133.

Ebenda, S. 127.

Ebenda, S. 127.

Torquemada, Jardin (Anm. 53), S. 175. Auch Antonio betont, daß die Artenvielfalt und das Vorkommen von Monstrositäten im Meer denen zu Lande und in der Luft entspreche, das Meer aber doch einige Besonderheiten, die es als Milieu von den anderen beiden unterscheiden, aufweise (S. 471). Kausal denkt Bernardo, der die unterschiedlichen Kategorien der Monster in verschiedenen Erdteilen beheimatet vermutet, und so wohl an ein mögliches regelmäßiges Gesetz 
Der Raum kann als Unterscheidungsmerkmal dienen, kann aber auch eine Ursache angeben. ${ }^{61}$ Den als bekannt vorstellbaren Örtlichkeiten stehen unbekannte gegenüber. Weitgehend unerforscht sind für die Gesprächspartner die nördlichen Regionen. Daher werden die Berichte darüber eingehenden Erörterungen unterzogen. Der Ort führt wie bei Huarte zur Differenz und zugleich zur Ursache für die Unterscheidung zwischen nördlichen und südlichen Menschen. Antonio glaubt, daß eine Ursache-Wirkung-Beziehung besteht zwischen dem Klima und der Größe der Menschen. Daher gebe es heute Riesen nur noch in der Arktis und Antarktis: "porque la naturaleza parece que se inclina a criar mayores hombres en las regiones más frías". ${ }^{62}$ Luis vermutet, daß große Menschen länger leben, da die Größe des Körpers der 'Größe' des Lebens entsprechen müsse. ${ }^{63}$ Derartige Analogieschlüsse werden von Antonio aber zugunsten kausalen Denkens strikt abgelehnt. Er führt stattdessen konkrete Ursachen wie eine bessere physische Ausstattung und moderatere Lebensweisen an: "por esas causas creo yo que viven tan larga vida algunas naciones de gentes". ${ }^{64}$ An anderer Stelle erläutert er weitere Ursachen dafür, daß die Menschen in den nördlichen Regionen länger leben: die reinere Luft, die vor Krankheiten schütze und die härteren Klimabedingungen, die den Körper robuster machten. ${ }^{65}$

Ist der Ort primär Mittel zur Klassifikation und erst sekundär für eine Ursachenangabe, dann ist die Zeit untrennbar mit Ursachenketten, aber auch, wie Aristoteles gezeigt hat, mit räumlicher Bewegung verbunden. Bernardo kann sich nicht erklären, warum die Länge bzw. Kürze der Tage im Verhältnis zwischen den letzten Breitengraden vor den Polen soviel schneller zunimmt als zwischen den näher dem Äquator gelegenen. Antonio kann dieses Problem mit seinem kausalen Denken erklären: "como esa tierra para con el sol se vaya siempre cuesta abajo, en poco espacio se encubre o descubre en mucha cantidad". ${ }^{66} \mathrm{Er}$

der Natur denkt: "a estos monstruos ¿ponen los autores en una parte o tierra juntamente, o en diveras partes?" (S. 127).

61 Antonio erwähnt z.B. die Dämonen, Schadensgeister, als deren Anführer Satan gilt, und teilt sie in sechs verschiedene Kategorien, da sie in jeweils unterschiedlichen Regionen von Himmel und Erde leben. Die ersten in der höchsten Gegend der Lüfte, die zweiten zwischen Luft und Erde, die dritten auf der Erde, die vierten im Wasser, die fünften in Höhlen und die sechsten in Abgründen. Je nach Aufenthaltsort haben sie ein unterschiedliches Wirkungsfeld, so daß die zweiten "fuera de la natural operación de naturaleza, mueven los vientos con mayor furia de la acostumbrada" (Torquemada, Jardin [Anm. 53], S. 254f.).

62 Ebenda, S. 160.

63 Sogar Theopompos wird zitiert, der sich in seinem Buch De Varia Historia eine andere Welt vorstellt, die außerhalb unserer Welt existiert und in der alles größer ist, wobei größere Menschen analog zu ihrer Größe auch über ein längeres Leben verfügen (ebenda, S. 416).

Ebenda, S. 161.

65 Vgl. ebenda, S. 429. Auf die Frage, wie die Menschen es schaffen, in den kalten Polarregionen zu überleben, wo doch in Spanien die Menschen schon bei viel geringeren Temperaturen manchmal erfrören, findet Bernardo einen logischen Grund in der allmählichen Anpassung des Menschen an die Umwelt, in der er aufwächst, d.h. in einem Prozeß der Entwicklung.

66 Torquemada, Jardín (Anm. 53), S. 408. Er unterlegt diese Erklärung noch dadurch, daß er die Erfahrung eines Wanderers wiedergibt, der die Sonne unten an einem Berg untergehen sieht und, falls er den Berg schnell besteigt, oben erkennt, daß die Sonne noch viel höher am Himmel steht. Weiter erklärt Antonio, daß analog zur Annäherung an die Pole (also an die der Sonne fernen Brcitengrade) die Tage immer länger bzw. je nach Jahreszeit kürzer werden, bis man zu dem 
schlägt vor, in einem Experiment mit einer Kerze und einer Kugel diese physikalischen Gegebenheiten zu überprüfen und herauszufinden, warum es auch während der Polarnacht nie stockdunkel ist. Der naturwissenschaftlichen Wirkursache hierfür fügt er als Zielursache hinzu, daß die Natur immer das Beste für den Menschen wolle. ${ }^{67}$

$\mathrm{Da} ß$ die Zeit zu ständigen Veränderungen in der Natur und bei den Menschen führt, erkennt Antonio, da sich viele von den Alten erwähnte Flüsse oder Länder heute nicht mehr ausfindig machen lassen, da Länder, Regionen etc. immer wieder umbenannt werden: "así como cada año se visten los árboles, las plantas y hierbas, y se despojan y tornan otra vez a renovarse, y mueren unos hombres y nacen otros y acaece lo mismo en los animales, aves y pescados y en todas las otras cosas, así acaece y sucede lo mismo en lo de los nombres de las mismas cosas". ${ }^{68}$ Auch die menschlichen Ängste und Leidenschaften, insofern sie von einer Ursache abhängig seien, sind für Antonio nicht einfach konstante Eigenschaften, sondern entwickelten sich geschichtlich entsprechend einer Veränderung der Ursachen: "así como la complexión, que es la causa, se puede mudar, y se muda muchas veces con el tiempo o con otras causas accidentadas, también se pueden mudar las que llamáis naturales inclinaciones, y pasiones, o impotencias [...] la edad y el tiempo y los accidentes muchas veces mudan unas complexiones en otras, y juntamente las condiciones y pasiones [...]". ${ }^{9}$

Es hat sich gezeigt, daß bei der Einbeziehung von Ort und Zeit aus der taxonomischen Betrachtungsweise nicht selten auch eine ursachenbezogene wurde. Explizit ausgehend von Aristoteles, jedoch christlichen Philosophen wie Thomas von Aquin den Vorzug gebend, läßt Torquemada seinen Protagonisten Antonio das Prinzip von natura naturans und natura naturata erläutern. Dieser Gegensatz ist erstmalig in der lateinischen Übersetzung des Kommentars des Averroes zu Aristoteles Schrift über den Himmel zu finden, ${ }^{70}$ wobei sich natura naturata auf das Weltganze in seinem geschöpflichen Verhältnis zu Gott als dem ersten Verursachenden, der natura naturans, bezieht. Da die Kausalkette also mit Gott beginnt, dürfe man bei keiner auch noch so wundersamen Erscheinung argumentieren, daß es sie nicht geben könne, da Gott alles erschaffen kann, ohne sich an Naturgesetze zu halten, die der. Verstand des Menschen erkannt zu haben glaubt: "el fundamento de donde todo procede, que es Dios." "71 Gerade deshalb hält er es für unangemessen, angesichts neuer und wundersamer Phänomene in Erstaunen zu verfallen und von der Vernunft abzusehen. ${ }^{72}$

Während beim letzten Beispiel eine Wirkursache angenommen wurde, wird ein ungewöhnliches Phänomen im folgenden durch eine Zielursache erklärt. Über die

Extrem in der Gegend um die Pole gelangt, wo sowohl die Nacht als auch der Tag jeweils ein halbes Jahr dauern.

67 "Que así como la naturaleza provee en el remedio de todas las cosas, porveyó en dar algún alivio para que no se sintiese con tanto trabajo en una noche tan larga como la de medio año" (Torquemada, Jardin [Anm. 53], S. 404).

Ebenda, S. 497.

Ebenda, S. 247f.

70 De caelo I, 1, 268a, 19.

71 Torquemada, Jardín (Anm. 53), S. 105.

72 Er bestreitet daher, daß Katastrophen auf der Erde wie die Pest von den Sternen erzeugt werden, da nichts ohne den Willen Gottes geschehe, aber auch durch konkrete, irdische Ursachen: "Y concluyendo, digo que las enfermedades pestilenciales se causan de cosas de la misma tierra, que son de los aires que pasan por donde hay algunos animales muertos y corrompidos y de las aguas detenidas que se corrompen e hieden, u otras cosas hediondas y dañosas" (ebenda, S. 379). 
wundersame Geschichte von einem Ziegenbock, dem wie einer Ziege Euter voller Milch wuchsen, bemerkt Antonio, daß von demselben Phänomen auch der Autor Andria Matiolo Senés berichtet, der dann herausfand, daß die Milch des von ihm untersuchten Ziegenbocks ein hervorragendes Mittel gegen Epilepsie war. Darauf bemerkt Bernardo: "No debió de faltar causa para que naturaleza saliese de su orden en una cosa como ésa, y por ventura, sería para poner algún remedio en una enfermedad que por tan incurabile se tiene."73 Der Ziegenbock erzeugt also Milch, um für den Menschen ein Mittel gegen Epilepsie hervorzubringen, womit das Phänomen rational erklärt ist. ${ }^{74}$

Immer wieder sind es rationale Ursachen, mit denen Außergewöhnliches und Wunderbares erklärt wird. So nimmt Bernardo einen Bericht über furchtbare Geräusche in einer bestimmten hohen Bergkette nicht einfach hin, sondern denkt über deren Ursache nach: "lo que se sospecha que puede causar esta maravilla es que hay algunas hendeduras y cuevas en las peñas de aquellos montes, y que el flujo y reflujo del agua que combate con el viento, el cual no tiene por dónde poder expirar, hace aquel son tan temeroso y espantable". ${ }^{75}$ Antonio entkräftet mit logisch-kausalem Denken Gerüchte, nach denen die Menschen auf der anderen Seite der Welt auf dem Kopf stünden: "Porque todas las cosas de la tierra naturalmente apetecen y quieren ir hacia bajo a buscar el centro de la tierra, adonde quiera que esté un hombre y en cualquier parte del mundo que es redondo [...]."76

Während im vorigen Fall eine zufriedenstellende Ursache gefunden werden konnte, muß der Protagonist bei folgendem wundersamen Phänomen zugeben, keine Lösung zu haben, ohne aber auszuschließen, daß es eine Ursache gibt. Mit dem Bericht von einer wundersamen Quelle konfrontiert, deren Wasser zu Stein erstarrt, sobald es auf den Boden fällt, ohne dann je wieder flüssig zu werden, schließt Luis in seiner Suche nach der Ursache des Phänomens die ihm wohlbekannte Gesetzmäßigkeit, daß Kälte Wasser zum Gefrieren bringt und dieses dann bei Hitze wieder auftaut, aus: "de donde se puede inferir que no es la frialdad la que hace esta dureza, sino alguna otra causa que a nosotros nos es oculta y que podría mal averiguarse". 77 Allzu einfache analoge Erklärungen jedenfalls werden erneut kritisiert. Antonio korrigiert an einer Stelle das analoge Denken Bernardos durch evolutivkausales Denken. Bernardo hatte die Steine in der Erde die "huesos de la tierra" da sie seiner Meinung nach genau wie die Knochen immer dieselbe Größe behielten. Antonio setzt dem entgegen, daß die Steine sich beständig verändern, wachsen oder kleiner werden. Wie sie sich entwickelten, in welcher Zeit sie hinsichtlich Quantität und Qualität zu- oder abnähmen, hinge vielmehr von ihrem Material, Ort und Umfeld ab. Ort und Zeit dienen also nicht nur der Differenzierung, sondern auch der Veranschaulichung von Ursachenketten.

73 Torquemada, Jardin (Anm. 53), S. 115.

74 Bei der Medizin jedenfalls ist das Vertrauen in die Möglichkeit, vernünftige Ursachen zu finden, ungebrochen. Antonio ist überzeugt, daß die Medizin einst Gründe finden wird für bislang noch unerklärliche Phänomene wie das, daß einige Menschen lange Zeit ohne zu trinken überleben können: "la razón dejémosla para los médicos, que darán causas suficientes para que entendamos cómo sea posible lo que tan fuera del orden natural parece" (ebenda, S. 153). Ebenda, S. 451.

Ebenda, S. $386 f$.

Ebenda, S. 203.

Ebenda. 
Auch bei der Unterscheidung zwischen Mensch und Tier oder zwischen Mann und Frau bedient sich Torquemada der aristotelischen Lehre von der Definition durch spezifische Differenz und nächstliegende Gattung, wie im folgenden deutlich wird, wo jedoch Ursachen, Genese und Entwicklung im Mittelpunkt stehen. So erklärt Luis, daß ein Mensch und ein Tier niemals zusammen Nachwuchs der einen, der anderen oder einer neuen Art zeugen können, sowie allgemein auch zwei verschiedene Tierarten dies nicht könnten, da ihre Anlagen zu verschieden seien. Dies sei nur ausnahmsweise bei solchen Tieren möglich, die nur durch wenige Merkmale voneinander getrennt sind. Er unterscheidet also die verschiedenen Tierarten durch ihre unterschiedlichen Merkmale und konstatiert Ähnlichkeit bei wenigen unterschiedlichen Merkmale und Unähnlichkeit bei vielen unterschiedlichen Merkmalen. Die Fortpflanzung von Hunden mit Wölfen oder Eseln mit Pferden erscheint jedenfalls möglich: "Porque con ser estos animales tan poco diferentes unos de otros, hace que la contradicción no sea tan grande como lo es cuando difieren en tantas cosas como difiere un hombre de los otros animales."79

Die spezifische Differenz zwischen Mensch und Tier ist bei Torquemada wie bei Aristoteles der Verstand. Gegen Luis, der der Meinung ist, auch Tiere haben Verstand, was man an den organisierten Gemeinschaften von Bienen, Ameisen und Kranichen sehe, setzt sich Antonio durch, für den der Mensch das einzige Wesen mit Verstand ist. Dies hindert ihn aber nicht, auch Tiere je nach mehr oder weniger ausgebildetem Instinkt zu unterscheiden. ${ }^{80}$ An anderer Stelle leitet er aus der Unterscheidung der Tiere nach der Komplexität ihrer Fähigkeiten eine Hierarchie der Tiere zu Land und zu Wasser ab. Deshalb überrascht ihn die Erzählung über eine Art Fischmenschen mit fast menschlichem Verstand nicht. ${ }^{81}$

Als nicht weniger einfach erweist sich die Differenzierung von Mann und Frau angesichts von Erzählungen über Abweichungen von der natürlichen Ordnung. Die aus der griechischen Mythologie bekannten gottähnlichen Hermaphroditen, die das maskuline und feminine Geschlecht in sich vereinigen, werden bei Torquemada zu Bewohnern von Burgos und Sevilla, von denen Luis und Antonio zu berichten wissen. Luis geht davon aus, daß viele Menschen mit einer weiblichen und einer männlichen Natur geboren werden, daß aber eine davon meist so schwach ausgeprägt ist, daß sic nicht auffällt. Er unterscheidet nun verschiedene Grade der Ausprägung und nennt das Beispiel eines Einwohners von Burgos, dem man, da er zugleich Mann und Frau war, unter Androhung der Todesstrafe auferlegt

79 Torquemada, Jardin (Anm. 53), S. 178.

80 Mit Bezug auf Albertus Magnus' De animalibus teilt er die Tiere in unterschiedliche Kategorien: "La prudencia y la sagacidad y la astucia de los animales brutos no están en unos más que en otros porque tengan algún entendimiento o razón en aquellas cosas que hacen, sino porque son mejor complexionados, y los sentidos están de mayor perfección, y también porque influyen mejor en ellos los cuerpos celestiales, para que mejor sea guiado su apetito por instinto y por naturaleza“ (ebenda, S. 348). Luis stellt fest, daß sich die Verhältnisse im Meer analog zu denen auf dem Land verhalten: „También en los pescados hay algunos de mayor instinto natural, y tanto, que casi parecen tener mayor entendimiento que otros, conforme a lo que vemos en los animales" (ebenda, S. 478).

81 “[...] así como vemos que acá hay algunos animales de mayor instinto natural y que están más propincuos a la razón y a contrahacer a los hombres, como son los simios, también en la mar habrá pescados que en esto se diferenciarán de los otros" (Torquemada, ebenda, S. 175). 
hatte, sich für eine Art zu entscheiden, und der verbrannt wurde, nachdem er sich als Mann betätigte, obwohl er sich für die Art der Frau entschieden hatte. Zwar wird auch Aristoteles angeführt, der nach Antonio von solchen Zwitterwesen berichte, "que estos andróginas tienen la teta derecha como hombre y la siniestra como mujer, porque con ella alimentan las criaturas que paren." ${ }^{, 82}$ Luis aber ist es, der als kausale Erklärung die Nebenursache des Orts anführt, wenn er erklärt: "A mí maravillado me tiene: y creo que alguna influencia o constelación que reina en esa provincia será causa de lo que habéis dicho, o propiedad de la misma tierra, que engendra las gentes de esa manera, como en otras tierras se engendran con diferentes condiciones y calidades. ",83

An dieser Stelle sei an den anfangs erwähnten aristotelischen Bockhirsch oder an die Rindsgattung mit Mannsbug erinnert, die Aristoteles dadurch erklärte, daß sie durch materiell oder formal entgegenstehende Ursachen an ihrer vollen Entwicklung gehindert wurden. Beim Versuch, der Ursache auf den Grund zu gehen, werden bei Torquemada ungewöhnliche Geburten und deren immer vorhandene, wenn auch nicht immer erkennbare Ursachen besprochen: "proceden, o de la voluntad y permisión del que todo lo tiene en su mano, o por algunas causas y razones a nosotros encubiertas". ${ }^{84}$ Danach bemüht er sich zu differenzieren, indem er zunächst von einer allgemeinen, natürlichen Regel ausgeht, von der er dann besondere Abweichungen aufzeigt und herausarbeitet, welche Merkmale diese haben. ${ }^{85}$ Dann werden weitere Arten ungewöhnlicher Geburten aufgezählt, z.B. daß Kinder schon mit Zähnen geboren werden, wobei innerhalb dieser Art noch Unterarten differenziert werden, solche z.B., die bei der Geburt nur die Vorderzähne haben, solche, die einen Knochen an der Stelle haben, und Herkules, der bei der Geburt drei Reihen von Zähnen hatte. Antonio führt die Aufzählung weiter fort, indem er die Art der Geburten, bei denen die Natur nur ein wenig von der allgemeinen Regel abweicht, verläßt, um mit der Art zu beginnen, wo die Abweichungen noch viel außergewöhnlicher sind: "Ésas son cosas en que la naturaleza parece salir poco de su orden concertada; y así, subamos a referir otras mayores y de mayor admiración." 86

Beim Bericht von einer Frau, die nur ein winziges, mißgeformtes Wesen gebar, sucht Bernardo nach einem Grund: "por ventura el defecto estuvo en el sujeto de la mujer, o en la simiente genital del padre, que con su imperfección no bastó a engendrar creatura más perfecta. ${ }^{87}$ Antonio und Luis wägen sogar verschiedenste Gründe $a b$, die die Ursache von Monstergeburten sein könnten: "en la simiente genital puede haber algunas superfluidades, que, corrompiéndose, en lugar de engendrar criatura, engendran esos animales [...] Pero más cierto es que se engendran de la superfluidad de los humores corrompidos que están en

85 "Natural cosa es todas las criaturas dar la vuelta en el vientre de su madre y venir a salir de él con la cabeza para adelante; pero muchas veces falta esta regla general, y algunos salen atravesados y el cuerpo doblado [...]. Otros, dejando de venir de cabeza, salen con los pies para adelante [...]" (ebenda, S. 118).

86 Ebenda, S. 120.

87 Ebenda, S. 121 
el cuerpo de la mujer [...]." ${ }^{, 88}$ Die These der überflüssigen, schädlichen Säfte im Körper der Frau als Ursache für Monstergeburten wird also teleologisch begründet. Die Monstergeburt nämlich sei notwendig gewesen, um die schlechten Säfte auszuscheiden, um der Frau Schmerzen zu ersparen und ihr Leben zu retten. Diese Überlegung folgt in Anlehnung an die Annahme des Aristoteles, daß in der Natur nie etwas ohne Grund geschieht, sondern die Natur innerhalb ihrer Möglichkeiten immer nach der besten Lösung strebt: "la naturaleza siempre se esfuerza a hacer de las cosas posibles lo que es mejor." ${ }^{89}$ Zudem zeigt sich hier erneut das aristotelische Erklärungsmuster für Bockhirsch oder Sphinx, nach dem materielle bzw. formale entgegenstehende Ursachen ein Wesen an der vollen Entwicklung hindern.

Zusammenfassend lässt sich festhalten, daß das aristotelische Modell einerseits der Differenzierung von Arten und Gattungen und andererseits der vier Ursachen der Veränderung nicht nur bei Philosophen wie Luis de Granada, Francisco Sánchez, Juan Huarte und Ginés de Sepúlveda, sondern auch beim humanistischen Schriftsteller Antonio de Torquemada eine zentrale Position einnimmt. So zeigt sich im Zusammenhang mit der im Spanien des 16. Jahrhunderts zentralen Aristotelesrezeption die Präsenz der Episteme von Differenz und Ursache, die Foucault erst für das 17. bzw. 19. Jahrhundert annimmt.

Ausblickend sei erwähnt, daß nach Aristoteles das Sich-Wundern zur Entstehung der Philosophie, nämlich zur Suche nach den Ursachen, z. B. nach der Entstehung des Alls, führt. ${ }^{90}$ Alle Wissenschaften nämlich, sagt Aristoteles, beginnen "mit der Verwunderung, daß die Dinge so sind, wie sie sind, wie etwa angesichts sich selbst bewegender Marionetten [gemeint sind die Figuren aus dem platonischen Höhlengleichnis], der Sonnenwende oder der Inkommensurabilität der Diagonale."91 Betrachtet man also nun als Ziel das philosophisch-wissenschaftliche Denken, dann ist es schon fast gleichgültig, ob es sich an Realem oder an wundersam Irrealem entwickelt. Wenn es Torquemada primär um die intellektuelle Erfassung der Welt geht, könnte er auch folgendem Satz des Aristoteles zustimmen: "So ist auch ein Liebhaber von Mythen in gewisser Hinsicht ein Philosoph, setzt sich doch ein Mythos aus Wunderbarem zusammen." ${ }^{92}$ Das Wunderbare des Monsters hätte dann nicht zuletzt den unbestreitbar heuristischen Vorzug, zur philosophischen Analyse von Taxonomien und Kausalketten zu führen, die bei Torquemada durchaus nicht nachgeordnet ist.

88 Torquemada, Jardin (Anm. 53), S. 121f. Luis nennt als weiteren möglichen Grund noch die Phantasien der Frau während der Empfängnis.

89 Ebenda, S. 122.

90 "Weil sie sich nämlich wunderten, haben die Menschen zuerst wie jetzt noch zu philosophieren begonnen" (Aristoteles, Metaphysik, hg. v. Franz F. Schwarz, Stuttgart 1970 [Reclam], S. 21; vgl. auch Platon, Theaitetos 155).

91 Aristoteles, Metaphysik, (Anm. 90), S. 23.

92 Ebenda, S. 22. 